\title{
Contribution of acoustic radiation force impulse (ARFI) elastography to the ultrasound diagnosis of biliary atresia
}

\author{
Sylviane Hanquinet ${ }^{1}$ - Delphine S. Courvoisier ${ }^{2} \cdot$ Anne-Laure Rougemont $^{3}$ • \\ Amira Dhouib $^{1}$ - Laura Rubbia-Brandt ${ }^{3}$ - Barbara E. Wildhaber ${ }^{4}$. \\ Laura Merlini $^{1}$ - Valerie A. McLin ${ }^{5}$ - Mehrak Anooshiravani ${ }^{1}$
}

Received: 19 November 2014 / Accepted: 1 April 2015 /Published online: 6 May 2015

(C) Springer-Verlag Berlin Heidelberg 2015

\begin{abstract}
Background Children with biliary atresia rapidly develop liver fibrosis secondary to inflammatory destruction of the biliary tract. Noninvasive detection of liver fibrosis in neonatal/ infantile cholestasis is an additional criterion for the diagnosis of biliary atresia, leading to prompt surgical exploration.

Objective To assess the value of US with acoustic radiation force impulse (ARFI) elastography to detect biliary atresia in the workup of neonatal/infantile cholestasis.

Materials and methods In this retrospective study, 20 children with cholestasis suspected of having biliary atresia were investigated by US and ARFI. We evaluated the association between US findings and the diagnosis of biliary atresia and with two scores of liver fibrosis obtained from liver biopsy. Results In univariate analyses, gallbladder size, triangular cord sign, spleen size and ARFI values were found to be associated with biliary atresia, though only the triangular cord sign remained significant when elevated gamma glutamyltransferase (GGT) was included as a predictor. In contrast, spleen size and
\end{abstract}

Sylviane Hanquinet

sylviane.hanquinet-ginter@hcuge.ch

1 Department of Pediatric Radiology, University Children's Hospital, 6 rue Willy Donzé, Ch 1211, Geneva 14, Switzerland

2 Division of Clinical Epidemiology, University of Geneva, Geneva, Switzerland

3 Division of Clinical Pathology, University Hospital of Geneva, Geneva, Switzerland

4 Department of Pediatric Surgery, Children's University Hospital of Geneva, Geneva, Switzerland

5 Department of Pediatric Gastroenterology, Children's University Hospital of Geneva, Geneva, Switzerland
ARFI correlated with the degree of liver fibrosis on biopsy $(r>$ $0.70, P<0.001$, which remained significant when gamma glutamyltransferase elevation was included as a predictor. Conclusion The addition of ARFI to a standard abdominal US in the initial workup of the neonate with possible infantile cholestasis can provide reliable information on liver fibrosis and help in the diagnosis of biliary atresia.

Keywords Acoustic radiation force impulse sonography . Biliary atresia $\cdot$ Cholestasis $\cdot$ Liver $\cdot$ Neonate $\cdot$ Shear wave elastography $\cdot$ Ultrasound

\section{Introduction}

The prevalence of neonatal cholestasis varies between $1 / 2,500$ and 1/5,000 live births. It presents in the first weeks of life by jaundice and pale stools.

Neonatal cholestasis may be secondary to various conditions such as biliary atresia, choledochal cyst, metabolic disease, Alagille syndrome, alpha 1 antitrypsin deficiency, cystic fibrosis and neonatal infections. The etiology in about $30 \%$ of all infants presenting with neonatal cholestasis is biliary atresia $[1,2]$. Prompt diagnosis is essential for timely surgical management because if untreated the disease rapidly progresses to liver fibrosis and cirrhosis [3-5]. At our center, intraoperative cholangiogram is the preferred final diagnostic approach, and a wedge- or needle-liver biopsy is obtained intraoperatively. Still, a tool to help determine which patients require surgical exploration is urgently needed.

At present, no single specific noninvasive diagnostic method can effectively differentiate the causes of cholestasis.

Ultrasonography is usually the first step in the workup of neonatal cholestasis $[6,7]$. To date, several sonographic criteria (gallblader size, triangular cord sign, splenomegaly, 
hepatic artery-portal vein ratio, hepatic subcapsular flow on color Doppler) may point to biliary atresia, but none is diagnostic [8]. Likewise, routine serum measurements are of limited sensitivity and specificity [9]. Hepatic fibrosis is the main complication of atresia, rapidly leading to cirrhosis. The gold standard detection of fibrosis is by biopsy, an invasive procedure. Noninvasive techniques for measuring liver fibrosis are poorly developed in children. They include biochemical tests such as APRI (aspartate transaminase-to-platelet ratio index), but results are inconclusive and vary widely across studies [10].

With these points in mind, it is of utmost importance to find complementary noninvasive methods to evaluate liver stiffness and liver damage, as surrogates for biliary atresia, in order to hasten the diagnosis of biliary atresia and shorten the time to intraoperative cholangiogram and Kasai operation, because prompt management is known to lead to favorable outcomes [3].

Pediatric studies on liver fibrosis evaluation by ARFI elastography are rare and mostly involve a mixture of liver diseases $[11,12]$. In the analysis of liver fibrosis in biliary atresia, one study reported experience with transient elastography [13], and another very recent study with 11 patients analyzed shear wave elastography by two ARFI methods - Virtual Touch Tissue Quantification (VTQ) and Virtual Touch Imaging Quantification (VTIQ) [14].

Our study assessed the value of US with ARFI elastography (VTQ) for a rapid diagnosis of liver fibrosis and for helping to differentiate biliary atresia from other causes of cholestasis.

\section{Materials and methods}

This study was approved by the institutional review board of our hospital. All patients were either born at our institution or referred from other centers between January 2011 and May 2014 with clinical suspicion of biliary atresia (acholic stools, jaundice and hepatomegaly). On admission to our hospital, all subjects underwent laboratory tests and abdominal US with liver stiffness measurement by ARFI. Seventeen children also had a liver biopsy and 13 children underwent intraoperative cholangiogram.

\section{Ultrasound and ARFI elastography}

The studies were all performed on an Acuson ${ }^{\circledR}$ S2000 or S3000 US machine (Siemens Healthcare, Erlangen, Germany) with a Virtual Touch Tissue Quantification (VTQ) mode for acoustic radiation force impulse (ARFI) elastography. ARFI measures the speed of a shear wave in the examined tissue in $\mathrm{m} / \mathrm{s}$. For abdominal US in babies, we used linear $9-\mathrm{MHz}$ and 14-MHz probes. ARFI measurements with a linear $9-\mathrm{MHz}$ probe were performed simultaneously with the abdominal US. The region of interest was placed in the right liver lobe, far from a visible vessel or bile duct, with an intercostal or subcostal approach, according to the best hepatic image accessibility.

Each child was measured five times and the mean was calculated. ARFI technique and reference values have been published [15]. Ultrasound and ARFI measurements were made by two senior pediatric radiologists (M.A. and S.H., each with 4 years of experience with the ARFI technique).

Ultrasound was the first investigation and thus was performed without knowledge of the laboratory results or histology. Patients fasted $4-6 \mathrm{~h}$ prior to the study. If the gallbladder was not seen, another US examination was performed after more than $6 \mathrm{~h}$ of fasting.

Based on previously published studies on biliary atresia, US criteria were systematically recorded: liver size (evaluated subjectively by the experienced radiologist, $\mathrm{SH}, 30$ years of experience; $\mathrm{MD}, 25$ years of experience), echostructure of the liver, biliary tract visibility, and spleen size (pole-to-pole diameter at the level of the spleen hilus); when the gallbladder was present, its length was measured; the triangular cord sign was searched and measured anteriorly, adjacent to the portal vein bifurcation; the hepatic artery and portal vein diameters were also measured and the hepatic artery-portal vein ratio was calculated. Other anatomical abdominal abnormalities were also recorded.

\section{Blood sample analysis}

The following laboratory values were obtained on each child upon admission to the hospital: conjugated bilirubin and total serum bilirubin, alkaline phosphatase (ALP), alanine aminotransferase (ALT), aspartate aminotransferase (AST), gamma glutamyltransferase (GGT), platelet count $\left(103 / \mathrm{mm}^{3}\right)$, prothrombin time/international normalized ratio (PT-INR).

Using these values, the aspartate-aminotransferase-toplatelet ratio (APRI) index was calculated in 19/20 children using $\mathrm{AST}=63 \mathrm{IU} / \mathrm{L}$ as the upper limit of normal at our institution.

\section{Liver biopsy analysis}

Surgical or needle core biopsies were routinely fixed in $10 \%$ buffered formalin and were paraffin-embedded. Liver biopsy evaluation was performed by one senior pathologist (A.-L.R. 20 years of experience in molecular pathology). For routine histological examination, $3-\mu \mathrm{m}$-thick sections were stained with hematoxylin-eosin (H\&E), with special stains consisting of Masson's trichrome for fibrosis evaluation, reticulin stain for liver architecture and fibrosis evaluation, and Perl's blue for hemosiderin deposition. A minimal biopsy length of $10 \mathrm{~mm}$ was required for histological assessment of needle biopsies. Two scoring systems were used: 
1) METAVIR score. This score has been validated for hepatitis $\mathrm{C}$ fibrosis staging in adults. It classifies fibrosis in five stages: F0, no portal fibrosis; F1, portal fibrosis without septa; F2, portal fibrosis with few septa; F3, portal fibrosis with numerous septa, no cirrhosis; and F4, cirrhosis.

2) The semiquantitave scoring system (SSS). This score assesses the portal tract fibrosis, number and width of septa, and also the severity of central vein and perisinusoidal fibrosis with a range from 0 (normal) to 37 (most severe fibrosis) [16].

\section{Statistical analysis}

Characteristics between biliary atresia and non biliary atresia groups were compared using the Fisher exact test for categorical variables and the Wilcoxon rank sum test for continuous variables. To examine whether sonographic parameters predicted biliary atresia status over and above biological parameters, we used multivariable logistic regression with biliary atresia status as the outcome and GGT as the strongest biological predictor, as well as additional ARFI measurement. The association of biological or US parameters with liver biopsy scores (METAVIR or SSS) was assessed using Spearman correlation. The use of Spearman correlation was necessary because biological and US parameters were not normally distributed. The Spearman correlation computes correlation using ranks instead of observed values, thus avoiding giving a large weight to outliers.

\section{Results}

Twenty-eight children were admitted to our institution for infantile cholestasis. Eight were excluded because jaundice was caused by unconjugated hyperbilirubinemia or other benign conditions, or because of missing data.
Of the 20 remaining children (10 girls), biliary atresia was confirmed in 10 using intraoperative cholangiogram and liver biopsy. Mean age at the time of examination (US with acoustic radiation force impulse [ARFI] elastography) was 52.1土 29.2 days and median was 34 days (Table 1 ). In biliary atresia cases, intraoperative cholangiogram by gallbladder puncture was obtained in four children and contrast injection showed intrahepatic biliary tract blockage; in six children the gallbladder was reduced to a fibrous tissue, which did not allow injection of contrast media. Six children had type 3 and four children had type 2 biliary atresia, according to the biliary atresia American surgical classification [17]. The children with biliary atresia were treated by surgical hepatoportoenterostomy (Kasai procedure). Two children had syndromic biliary atresia (polysplenia, malrotation, median liver, situs inversus, preduodenal portal vein, absent vena cava).

In the non biliary atresia group, the diagnoses were giant cell hepatitis $(n=5)$, common bile duct stone $(n=1)$, choledocal cyst $(n=1)$, cystic fibrosis $(n=1)$, Alagille syndrome $(n=1)$ and unknown cause $(n=1)$. Outcome was favorable in nine children, and one death occurred at 7 months old (cystic fibrosis) from severe hepatic failure. In the non biliary atresia group, cholangiography was performed in three children with initial clinical suspicion of biliary atresia.

\section{Biological tests and ultrasound findings (Table 2)}

Overall, all blood tests were abnormal in both groups, with significantly higher levels in children with biliary atresia except for ALP and PT-INR. This difference was particularly noticeable for GGT (non biliary atresia, 198.6 UI/l; biliary atresia, 535.2 UI/1; $P=0.002$ ).

On sonography the liver was increased in size in all children. Liver appearance was more often heterogeneous in the biliary atresia group, including the presence of hyperechoic nodules in two children. Prominent periportal echogenicity throughout the liver parenchyma was observed in all biliary atresia cases [18].

Table 1 Patient characteristics

\begin{tabular}{llll}
\hline & No biliary atresia $(n=10)$ & Biliary atresia $(n=10)$ & $P$-value \\
\hline Gender & & & 1.00 \\
$\quad$ Female & $5(50.0)$ & $5(50.0)$ & \\
$\quad$ Male & $5(50.0)$ & $5(50.0)$ & 1.00 \\
Prematurity (<36 weeks) & $2(20.0)$ & $1(10.0)$ & \\
Characteristics at time of study & Mean (SD) & Mean (SD) & 0.85 \\
Age (days) & $55.5(33.9)$ & $48.8(25.0)$ & 0.38 \\
Height (z-score) & $-1.9(2.0)$ & $-1.5(2.6)$ & 0.19 \\
Weight (z-score) & $-1.8(1.3)$ & $-1.0(1.0)$ & \\
\hline
\end{tabular}

$S D$ standard deviation 
Table 2 Biological and US data among children with and without biliary atresi. Values are expressed as mean (standard deviation)

\begin{tabular}{llll}
\hline & No biliary atresia & Biliary atresia & $P$-value \\
\hline Biological measurements & & & \\
Total serum bilirubin $(\mu \mathrm{mol} / \mathrm{l})$ & $111.1(118.0)$ & $158.7(36.7)$ & 0.01 \\
Conjugated bilirubin $(\mu \mathrm{mol} / \mathrm{l})$ & $60.6(69.4)$ & $88.6(21.9)$ & 0.03 \\
AST $(\mathrm{U} / \mathrm{l})$ & $168.7(247.7)$ & $303.9(225.1)$ & 0.007 \\
ALT $(\mathrm{U} / \mathrm{l})$ & $140.7(282.5)$ & $198.8(236.5)$ & 0.02 \\
GGT $(\mathrm{U} / \mathrm{l})$ & $198.6(130.4)$ & $535.2(311.7)$ & 0.002 \\
ALP $(\mathrm{U} / \mathrm{l})$ & $496.3(376.6)$ & $539.2(249.7)$ & 0.33 \\
PT-INR & $1.6(1.8)$ & $1.2(0.2)$ & 0.08 \\
Platelet count $(\mathrm{G} / \mathrm{l})$ & $510.1(219.4)$ & $525.0(241.8)$ & 0.93 \\
APRI & $0.6(0.5)$ & $1.0(0.5)$ & 0.053 \\
Ultrasound measurements & & & \\
ARFI velocity $(\mathrm{m} / \mathrm{s})$ & $1.7(0.6)$ & $2.2(0.4)$ & 0.049 \\
Spleen size $(\mathrm{mm})$ & $48.3(11.2)$ & $54.3(10.5)$ & 0.17 \\
Gallbladder size $(\mathrm{mm})$ & $16.9(8.6)$ & $9.0(6.0)$ & 0.02 \\
Hepatic artery/portal vein ratio & $0.4(0.1)$ & $0.4(0.1)$ & 0.13 \\
Triangular cord sign positive, $n(\%)$ & $1.0(10 \%)$ & $7(70 \%)$ & 0.02 \\
\hline
\end{tabular}

$A L P$ alkaline phosphatase, $A L T$ alanine aminotransferase, $A P R I$ aspartate transaminase-to-platelet ratio index, $A R F I$ acoustic radiation force impulse, $A S T$ aspartate aminotransferase, $G G T$ gamma glutamyltransferase, $H A$ hepatic artery, $P$ T-INR prothrombin time/international normalized ratio, $P V$ portal vein, $T C$ triangular cord
We also sought other US findings suggestive of biliary atresia described in the literature. We identified the pathological triangular cord sign in $7 / 10$ children with biliary atresia (mean thickness $4.0 \mathrm{~mm}$; range $2.7-6.6 \mathrm{~mm}$ ) and once in the non biliary atresia group $(P=0.02)$. Gallbladder length was significantly smaller in the biliary atresia group, and no gallbladder was seen in four children with biliary atresia. In five cases, it was very small $(\leq 13 \mathrm{~mm})$, and in one case it was apparently normal $(24 \mathrm{~mm})$. In the non biliary atresia group, the gallbladder was always seen. Spleen size and hepatic artery-portal vein ratio were not significantly different between the groups (Table 3). The child with the choledochal cyst had a 2-week history of jaundice, pale stools and clinical hepatomegaly. Ultrasonography showed a normal gallbladder and visibility of intrahepatic biliary tract. Cholangiography was performed to rule out biliary atresia and showed a choledochal cyst.

Among US criteria, the triangular cord sign was the best predictor of biliary atresia when associated with GGT values $(P=0.046)$

\section{Liver biopsy results and correlation with other signs}

The degree of liver fibrosis as assessed by the METAVIR score was variable, mainly in the non biliary atresia group, in whom the METAVIR scores ranged from F0 (no portal tract fibrosis) to F4 (cirrhosis). In the biliary atresia group, all infants showed at least an F2 (portal fibrosis with few septa) degree of fibrosis. The SSS scores ranged between 0 and 17 in the non biliary atresia group, and 5 or higher in the biliary atresia group. In the group with cholestasis from causes other than biliary atresia, the child who had high liver fibrosis values had giant cell hepatitis.

Among the biological markers, ALT, GGT, PT-INR and APRI were moderately associated with the METAVIR score, and only the platelet count was correlated with the SSS score (Table 4).

Spleen size was moderately to strongly correlated with the METAVIR score $(0.72 ; P=0.001)$ and the SSS score $(0.59$; $P=0.02$ ) (Table 4 and Fig. 1).

\section{ARFI results}

In the non biliary atresia group, mean ARFI value was $1.7 \mathrm{~m} / \mathrm{s}$ (SD, 0.6; median, 1.41; minimum, 1.12; maximum, 3.09). In this group there were, however, three cases in whom ARFI values were $\geq 2 \mathrm{~m} / \mathrm{s}$ (two cases of giant cell hepatitis and one case of Alagille syndrome). In the biliary atresia group, mean ARFI value was $2.2 \mathrm{~m} / \mathrm{s}$ (SD, 0.4; median, 2.07; minimum, 1.57; maximum, 2.94) (Table 3).

The Spearman correlation of ARFI measurements with liver biopsy was significant for both scoring systems: META VIR, $0.54(P=0.02)$ and SSS, $0.71(P=0.001)$ (Table 4).

\section{Discussion}

Among the causes of neonatal/infantile cholestasis, biliary atresia is a surgical emergency warranting prompt intervention 
Table 3 Detailed set of US data and fibrosis tests for each child

\begin{tabular}{|c|c|c|c|c|c|c|c|c|}
\hline $\begin{array}{l}\text { Biliary } \\
\text { atresia }\end{array}$ & $\begin{array}{l}\text { Corrected age } \\
\text { (days) }\end{array}$ & GGT & $\begin{array}{l}\text { METAVIR } \\
\text { score }\end{array}$ & $\begin{array}{l}\mathrm{SSS}^{\mathrm{b}} \\
\text { score }\end{array}$ & $\begin{array}{l}\text { Spleen diameter } \\
(\mathrm{mm})\end{array}$ & $\begin{array}{l}\text { Triangular } \\
\text { cord }(\mathrm{mm})\end{array}$ & $\begin{array}{l}\text { Hepatic artery/ } \\
\text { portal vein ratio }\end{array}$ & $\begin{array}{l}\text { ARFI velocity } \\
(\mathrm{m} / \mathrm{s})\end{array}$ \\
\hline- & 52 & 160 & - & - & 40 & - & 0.36 & 1.66 \\
\hline- & 11 & 134 & - & - & 54 & - & 0.50 & 1.34 \\
\hline- & 30 & 158 & - & - & 45 & 1.5 & 0.28 & 1.41 \\
\hline- & 61 & 123 & F0 & 0 & 43 & - & 0.41 & 1.20 \\
\hline- & 30 & 124 & F0 & 0 & 42 & - & 0.25 & 1.12 \\
\hline- & 32 & 452 & F0 & 1 & 44 & - & 0.38 & 2.00 \\
\hline - & 18 & 413 & $\mathrm{~F} 1$ & 1 & 30 & - & 0.25 & 1.57 \\
\hline - & 68 & 153 & F3 & 5 & 63 & - & 0.42 & 1.69 \\
\hline - & 60 & 51 & F3 & 4 & 66 & - & 0.51 & 2.26 \\
\hline - & 32 & 218 & F4 & 17 & 56 & - & 0.22 & 3.09 \\
\hline+ & 9 & 357 & $\mathrm{~F} 2$ & 5 & 48 & 3.7 & 0.51 & 1.57 \\
\hline+ & 33 & 319 & $\mathrm{~F} 2$ & 4 & 50 & 3.6 & 0.3 & 1.60 \\
\hline+ & 63 & 328 & $\mathrm{~F} 2$ & 6 & 60 & - & 0.58 & 2.46 \\
\hline+ & 49 & 640 & F3 & 7 & PS & 2.7 & 0.56 & 2.06 \\
\hline+ & 68 & 894 & F3 & 5 & 65 & - & 0.44 & 2.16 \\
\hline+ & 19 & 169 & F3 & 4 & PS & 6.6 & 0.45 & 2.27 \\
\hline+ & 66 & 267 & F4 & 15 & 66 & 4.4 & 0.37 & 1.95 \\
\hline+ & 35 & 619 & F4 & 14 & 54 & - & 0.23 & 2.07 \\
\hline+ & 8 & 594 & $\mathrm{~F} 4$ & 16 & 58 & 3.9 & 0.45 & 2.40 \\
\hline+ & 55 & 1165 & F4 & 16 & 64 & 3.1 & 0.45 & 2.94 \\
\hline
\end{tabular}

ARFI acoustic radiation force impulse, GGT gamma glutamyltransferase, $P S$ polysplenia

${ }^{a}$ The METAVIR score classifies histopathological fibrosis in five stages: F0, no portal fibrosis; F1, portal fibrosis without septa; F2, portal fibrosis with few septa; F3, portal fibrosis with numerous septa, no cirrhosis; and F4, cirrhosis

${ }^{\mathrm{b}}$ The semiquantitave scoring system (SSS) assesses the portal tract fibrosis, number and width of septa, and also the severity of central vein and perisinusoidal fibrosis. The final score is calculated: $\mathrm{SSS}=\mathrm{CLV}+\mathrm{PS}+\mathrm{PT}+2(\mathrm{NSxWS})$ and ranges from 0 to 37

to improve prognosis [5]. Liver fibrosis is a severe complication of biliary atresia and prognosis depends on the degree of liver fibrosis at the time of Kasai procedure [19].

In our retrospective study of 20 children with suspected biliary atresia, we evaluated noninvasive methods to improve the preoperative diagnostic yield of biliary atresia. We focused on the contribution of noninvasive ultrasound ARFI elastography to detect liver fibrosis as validated by biopsy (gold standard) and thus provide an additional criterion for biliary atresia.

Ultrasound is a first-line investigation tool in neonatal/ infantile cholestasis. US criteria suggesting biliary atresia in the literature are the following: absent or small gallbladder, triangular cord sign and hepatic artery-portal vein diameter ratio [8, 20,21]. Although some authors describe the role of hepatic subcaspular flow on color Doppler [22], we did not record this sign in our study.

Among US findings that correlated with biliary atresia, we observed that spleen size correlated positively with the degree of fibrosis (METAVIR and ARFI) in children with biliary atresia [23]. By contrast, the triangular cord sign or hepatic artery-portal vein ratio did not correlate with the degree of fibrosis, confirming the findings of Shin et al. [13].

To our knowledge, only two studies have reported the contribution of elastography findings in this disease $[13,14]$. Our purpose was to confirm the reliability of ARFI in the diagnosis of biliary atresia in neonatal/infantile cholestasis. Indeed, we have previously shown that the mean ARFI value in the liver of healthy children is $1.12 \mathrm{~m} / \mathrm{s}$ (range $0.73-1.45 \mathrm{~m} / \mathrm{s}$ ) [15]. We have also demonstrated the reliability of ARFI in chronic liver disease in children, having calculated cut-off values compared to biopsy results. A cut-off of $1.34 \mathrm{~m} / \mathrm{s}$ detected $100 \%$ of children with fibrosis stage $>\mathrm{F} 0$, and a cut-off of $2.0 \mathrm{~m} / \mathrm{s}$ provided a sensitivity of $100 \%$ to detect SSS $>4$ or META $\mathrm{VIR}>\mathrm{F} 2$ in a pediatric population [12].

In our present study, we evaluated the contribution of ARFI elastography in assessing the degree of liver fibrosis in children with neonatal/infantile cholestasis based on a correlation with two histological scoring systems, METAVIR and SSS. ARFI elastography predicted the presence of biliary atresia with a mean propagation speed of $1.7 \pm 0.6 \mathrm{~m} / \mathrm{s}$ in non biliary atresia and $2.2 \pm 0.4 \mathrm{~m} / \mathrm{s}$ in biliary atresia. However, we 
Table 4 Spearman correlation of biological and US measurements with liver biopsy results

\begin{tabular}{|c|c|c|c|c|}
\hline \multirow[b]{2}{*}{ Biological measurement } & \multicolumn{2}{|l|}{ METAVIR $^{\mathrm{a}}$} & \multicolumn{2}{|l|}{$\mathrm{SSS}^{\mathrm{b}}$} \\
\hline & Correlation coefficient & $P$-value & Correlation coefficient & $P$-value \\
\hline Total serum bilirubin & 0.43 & 0.06 & 0.21 & 0.41 \\
\hline Conjugated bilirubin & 0.34 & 0.14 & 0.06 & 0.83 \\
\hline AST & 0.44 & 0.054 & 0.05 & 0.84 \\
\hline ALT & 0.48 & 0.03 & 0.07 & 0.80 \\
\hline GGT & 0.48 & 0.03 & 0.47 & 0.06 \\
\hline ALP & 0.11 & 0.66 & 0.35 & 0.16 \\
\hline PT-INR & 0.48 & 0.04 & 0.32 & 0.21 \\
\hline Platelet count & -0.21 & 0.40 & -0.49 & 0.049 \\
\hline APRI & 0.48 & 0.04 & 0.33 & 0.19 \\
\hline \multicolumn{5}{|l|}{ Ultrasound measurement } \\
\hline ARFI & 0.54 & 0.02 & 0.71 & 0.001 \\
\hline Spleen size & 0.72 & 0.001 & 0.59 & 0.02 \\
\hline Gallbladder size & -0.31 & 0.19 & -0.21 & 0.42 \\
\hline Hepatic artery/portal vein ratio & 0.03 & 0.90 & 0.11 & 0.67 \\
\hline Triangular cord sign positive & 0.30 & 0.22 & 0.37 & 0.15 \\
\hline
\end{tabular}

$A L P$ alkaline phosphatase, $A L T$ alanine aminotransferase, $A P R I$ aspartate transaminase-to-platelet ratio index, $A R F I$ acoustic radiation force impulse, $A S T$ aspartate aminotransferase, GGT gamma glutamyltransferase, $P T$ $I N R$ prothrombin time/international normalized ratio

${ }^{a}$ The METAVIR score classifies histopathological fibrosis in five stages: F0, no portal fibrosis; F1, portal fibrosis without septa; F2, portal fibrosis with few septa; F3, portal fibrosis with numerous septa, no cirrhosis; and F4, cirrhosis

b The semiquantitave scoring system (SSS) assesses the portal tract fibrosis, number and width of septa, and also the severity of central vein and perisinusoidal fibrosis. The final score is calculated: SSS $=\mathrm{CLV}+\mathrm{PS}+\mathrm{PT}+$ 2(NSxWS) and ranges from 0 to 37 observed a slight overlap between the groups. Our results, with a larger number of patients, agree with the conclusions by Leschied et al. [14] that ARFI may help in the noninvasive differentiation of biliary atresia from other causes of neonatal/infantile cholestasis [14]. However we report three infants without biliary atresia who also presented pathological ARFI values. Larger studies are therefore needed in this domain.

Because liver fibrosis in biliary atresia is progressive, we may ask the question of the age at which it is detectable by ARFI, especially in neonates. In our study, both groups of patients had similar mean ages (39.4 days and 40.5 days).
Among them, an 8-day-old with biliary atresia had significant fibrosis on biopsy, with elevated ARFI values $(2.4 \mathrm{~m} / \mathrm{s})$, and a second neonate, age 9 days, had an ARFI value of $1.57 \mathrm{~m} / \mathrm{s}$. ARFI values may already be pathological early in life, like in our two cases, as soon as the child is suspected of having biliary atresia, but this must be confirmed with a larger series. We must, however, bare in mind that this may not always be the case because liver fibrosis in biliary atresia is progressive in the first weeks of life.

In our series biological parameters were very good predictors of biliary atresia status, the assay of GGT serum levels being the best predictor [9]. GGT is usually high in biliary
Fig. 1 ARFI velocities by METAVIR (histopathological fibrosis) score for non biliary atresia (non BA) and biliary atresia (BA). $F 0$ no portal fibrosis, $F 1$ portal fibrosis without septa, $F 2$ portal fibrosis with few septa, $F 3$ portal fibrosis with numerous septa, F4 cirrhosis, $A R F I$ acoustic radiation force impulse

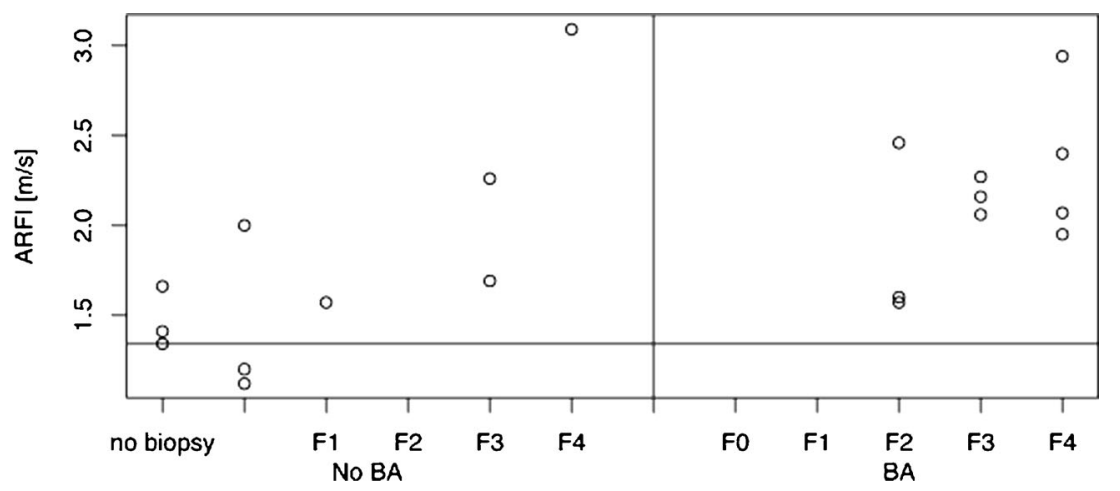


atresia, although the level varies with age, as explained by Rendón-Macías et al. [24].

US parameters were better predictors of biliary atresia status. Only the triangular cord sign remained informative of biliary atresia status when controlling for GGT levels. ARFI elastography was a noninvasive method to detect liver fibrosis. In particular, spleen size together with ARFI elastography were correlated with METAVIR and SSS scores.

It is interesting to note that in our series of 10 children with biliary atresia, 4 had pathological ARFI values without the triangular cord sign or with a normal gallbladder. This suggests the hypothesis that elastography may contribute to the diagnosis of biliary atresia by identifying liver fibrosis.

The main limitation of our study is the small sample size; biliary atresia is relatively rare and we only selected infants with ARFI measurements, a technique used at our institution since 2010. Because every medical center has few cases of biliary atresia, it would be interesting to gather more data in a multicenter study.

\section{Conclusion}

It is a challenging and fundamental question to distinguish biliary atresia from other causes of cholestasis in the neonatal/infantile population because surgical intervention is essential before the age of 3 months in order to improve prognosis. The first investigation of neonatal/infantile cholestasis should combine abdominal US with liver elastography to provide information on liver fibrosis. The present study demonstrates that ARFI elastography is a reliable complementary US technique to diagnose biliary atresia in infants presenting with cholestasis.

Acknowledgments The authors thank Isabelle Bosquet, nurse of pediatric radiology Children's Hospital Geneva, Switzerland, for data management.

\section{Conflicts of interest None}

\section{References}

1. Fischler B, Lamireau T (2014) Cholestasis in the newborn and infant. Clin Res Hepatol Gastroenterol 38:263-267

2. Baumann U, Ure B (2012) Biliary atresia. Clin Res Hepatol Gastroenterol 36:257-259

3. Chardot C, Buet C, Serinet MO et al (2013) Improving outcomes of biliary atresia: French national series 1986-2009. J Hepatol 58: 1209-1217

4. Nakamura K, Tanoue A (2013) Etiology of biliary atresia as a developmental anomaly: recent advances. J Hepatobiliary Pancreat Sci 20:459-464
5. Serinet MO, Wildhaber BE, Broue P et al (2009) Impact of age at Kasai operation on its results in late childhood and adolescence: a rational basis for biliary atresia screening. Pediatrics 123:1280 1286

6. Takamizawa S, Zaima A, Muraji T et al (2007) Can biliary atresia be diagnosed by ultrasonography alone? J Pediatr Surg 42:20932096

7. Donia AE, Ibrahim SM, Kader MS et al (2010) Predictive value of assessment of different modalities in the diagnosis of infantile cholestasis. J Int Med Res 38:2100-2116

8. Mittal V, Saxena AK, Sodhi KS et al (2011) Role of abdominal sonography in the preoperative diagnosis of extrahepatic biliary atresia in infants younger than 90 days. AJR Am J Roentgenol 196:W438-W445

9. Shah I, Bhatnagar S, Dhabe H (2012) Clinical and biochemical factors associated with biliary atresia. Trop Gastroenterol 33:214217

10. Diaz JJ, Gura KM, Roda J et al (2013) Aspartate aminotransferase to platelet ratio index correlates with hepatic cirrhosis but not with fibrosis in pediatric patients with intestinal failure. J Pediatr Gastroenterol Nutr 57:367-371

11. Noruegas MJ, Matos H, Goncalves I et al (2012) Acoustic radiation force impulse-imaging in the assessment of liver fibrosis in children. Pediatr Radiol 42:201-204

12. Hanquinet S, Rougemont AL, Courvoisier D et al (2013) Acoustic Radiation Force Impulse (ARFI) elastography for the noninvasive diagnosis of liver fibrosis in children. Pediatr Radiol 43:545-551

13. Shin NY, Kim MJ, Lee MJ et al (2014) Transient elastography and sonography for prediction of liver fibrosis in infants with biliary atresia. J Ultrasound Med 33:853-864

14. Leschied JR, Dillman JR, Bilhartz J et al (2015) Shear wave elastography helps differentiate biliary atresia from other neonatal/infantile liver diseases. Pediatr Radiol 45:366-375

15. Hanquinet S, Courvoisier D, Kanavaki A et al (2013) Acoustic radiation force impulse imaging-normal values of liver stiffness in healthy children. Pediatr Radiol 43:539-544

16. Chevallier M, Guerret S, Chossegros P et al (1994) A histological semiquantitative scoring system for evaluation of hepatic fibrosis in needle liver biopsy specimens: comparison with morphometric studies. Hepatology 20:349-355

17. Hartley JL, Davenport M, Kelly DA (2009) Biliary atresia. Lancet 374:1704-1713

18. Tan Kendrick AP, Phua KB, Ooi BC et al (2003) Biliary atresia: making the diagnosis by the gallbladder ghost triad. Pediatr Radiol 33:311-315

19. Wildhaber BE, Coran AG, Drongowski RA et al (2003) The Kasai portoenterostomy for biliary atresia: a review of a 27 -year experience with 81 patients. J Pediatr Surg 38:1480-1485

20. Jiang LP, Chen YC, Ding L et al (2013) The diagnostic value of high-frequency ultrasonography in biliary atresia. Hepatobiliary Pancreat Dis Int 12:415-422

21. Humphrey TM, Stringer MD (2007) Biliary atresia: US diagnosis. Radiology 244:845-851

22. El-Guindi MA, Sira MM, Konsowa HA et al (2013) Value of hepatic subcapsular flow by color Doppler ultrasonography in the diagnosis of biliary atresia. J Gastroenterol Hepatol 28:867-872

23. Liu P, Li P, He Wet al (2009) Liver and spleen volume variations in patients with hepatic fibrosis. World J Gastroenterol 15:3298-3302

24. Rendón-Macías ME, Villasís-Keever MA, Castañeda-Muciño G et al (2008) Improvement in accuracy of gamma-glutamyl transferase for differential diagnosis of biliary atresia by correlation with age. Turk J Pediatr 50:253-259 